\title{
Desarrollo de inteligencias múltiples usando tecnologías
}

\author{
Development of multiple intelligences using technologies
}

Desenvolvimento de inteligências múltiplas usando tecnologias

Segundo Vicente Echeverría Desiderio

segundo.echeverriad@ug.edu.ec

https://orcid.org/0000-0002-0235-190X

Universidad de Guayaquil, Guayaquil-Ecuador

Digna Rocío Mejía Caguana

digna.mejiac@ug.edu.ec

https://orcid.org/0000-0002-4390-0522

Universidad de Guayaquil, Guayaquil-Ecuador
William Lenin Chenche Jácome

william.chenchej@ug.edu.ec

https://orcid.org/0000-0003-4258-2582

Universidad de Guayaquil, Guayaquil-Ecuador

Jaime Gabriel Espinosa Izquierdo

jaime.espinosai@ug.edu.ec

https://orcid.org/0000-0001-6842-8626

Universidad de Guayaquil, Guayaquil-Ecuador

Artículo recibido 13 de octubre 2021, arbitrado y aceptado 16 de noviembre 2021 y publicado 28 de enero 2022

\section{RESUMEN}

El objetivo del estudio fue determinar el uso de tecnologías para promover el desarrollo de las inteligencias múltiples en los estudiantes la carrera de Literatura de la Facultad de Filosofía, Letras y Ciencias de la Educación. Se realizó una investigación cuantitativa, tipo descriptiva, exploratoria, con diseño de campo, transversal, con una población de 120 estudiantes, aplicando un cuestionario. Como resultados se encontraron que los docentes promueven siempre el uso de tecnologías para el desarrollo de la inteligencia lingüística e interpersonal; medianamente, para la intrapersonal y la visual-espacial; y nunca para las lógico-matemática, corporal-cinética, musical y naturalista. Se concluyó que el uso de tecnologías en educación superior representa una oportunidad para el perfeccionamiento del potencial de los estudiantes; estas al ser utilizadas de forma eficiente por los docentes pueden potenciar el desarrollo de las diferentes inteligencias múltiples.

Palabras clave: Inteligencias múltiples; desarrollo de inteligencias; tecnologías; educación; literatura

\section{ABSTRACT}

The objective of the study was to determine the use of technologies to promote the development of multiple intelligences in the students of the Literature course of the Faculty of Philosophy, Letters and Educational Sciences. A quantitative, descriptive, exploratory, exploratory, cross-sectional, quantitative research was conducted with a population of 120 students, applying a questionnaire. The results showed that teachers always promote the use of technologies for the development of linguistic and interpersonal intelligence; moderately, for intrapersonal and visual-spatial intelligence; and never for logical-mathematical, bodilykinesthetic, musical and naturalistic intelligence. It was concluded that the use of technologies in higher education represents an opportunity for the improvement of students' potential; when used efficiently by teachers, they can enhance the development of the different multiple intelligences.

Key words: Multiple intelligences; development of intelligences; technologies; education; literature

\section{RESUMO}

O objetivo do estudo era determinar o uso de tecnologias para promover o desenvolvimento de múltiplas inteligências em estudantes da Faculdade de Filosofia, Literatura e Ciências da Educação. Foi realizada uma pesquisa quantitativa, descritiva, exploratória, de corte transversal e quantitativa com uma população de 120 estudantes, aplicando um questionário. Os resultados mostraram que os professores sempre promovem o uso da tecnologia para o desenvolvimento da inteligência lingüística e interpessoal; moderadamente para a inteligência intrapessoal e visual-espacial; e nunca para a inteligência lógico-matemática, corpórea-cinestésica, musical e naturalista. Concluiu-se que o uso de tecnologias no ensino superior representa uma oportunidade para a melhoria do potencial dos estudantes; quando usadas eficientemente pelos professores, elas podem melhorar o desenvolvimento das diferentes inteligências múltiplas.

Palavras-chave: Inteligências múltiplas; desenvolvimento de inteligências; tecnologias; educação; literatura 


\section{INTRODUCCIÓN}

Actualmente existe una tendencia en la educación en todos los niveles, dirigida a desarrollar las distintas maneras de aprender que poseen los estudiantes; por tanto, en esta investigación se trabajó con el desarrollo de las inteligencias múltiples mediante el uso de tecnologías de información y comunicación (TIC), considerando el hecho de que a nivel universitario también es posible conseguirlo, tal como lo plantea Gardner (1997, p.378) "los individuos continúan desarrollándose durante toda su vida”.

Según expresa Armstrong (2000), Gardner sugirió que la inteligencia trata sobre dos aspectos esenciales a ser considerados en la educación, como son: la posibilidad para solventar situaciones problemáticas; y la de generar productos en un ambiente abundante en distintos escenarios, naciendo así el constructo teórico de las inteligencias múltiples (IM), luego de adoptar esta concepción amplia y pragmática, el concepto de inteligencia adquiere una matiz funcional que se desarrolla en la vida de los individuos de maneras diversas; para ello Gardner aportó un modelo para delinear las capacidades del ser humano agrupándolas en ocho inteligencias, estas son: lingüística, lógico matemática, espacial, cinético-corporal, musical, interpersonal, intrapersonal y naturalista.

La inteligencia lingüística, es definida como la capacidad de utilizar las palabras de forma eficaz, tanto por escrito como oralmente, incluye la utilización adecuada de la sintaxis, la fonología, la semántica, y los usos prácticos del lenguaje, tales como la retórica, la mnemotecnia, la explicación y el metalenguaje. Por su parte, la lógico matemática, es concebida como la capacidad para el uso de los números con eficacia y de los procesos de razonamiento; incluye la sensibilidad a patrones y relaciones lógicas, afirmaciones y proposiciones y otras abstracciones relacionadas.

Con relación a la inteligencia espacial, se tiene que esta es definida como la capacidad de percibir el mundo de manera tanto visual como espacial de forma sensible y de ejecutar acciones o tareas basadas en ese aforo; está vinculado a la percepción del color, las líneas, la forma de los objetos, el espacio y las relaciones entre estos, también tiene que ver con la forma de representar detalladamente imágenes visuales, así como de orientarse eficazmente. La inteligencia cinético-corporal, se concibe como el dominio del cuerpo para expresar ideas y sentimientos, y facilidad para utilizarlo en la creación o transformación de objetos, incluye aquellas destrezas físicas tales como coordinación motriz, equilibrio corporal, destreza al realizar movimientos particulares, aplicación de fuerza, flexibilidad a la hora de realizar movimientos y velocidad al desplazarse.

Por su parte, la inteligencia musical, se define como la posibilidad especial que se tiene para escuchar, convertir y expresar las formas musicales, está relacionado con los elementos físicos propios del sonido tales como, la intensidad, tono, timbre, ritmo y melodía de una pieza musical. AulaPlaneta (2021a) plantea los beneficios del desarrollo de esta inteligencia en las personas, entre ellos destaca: favorece la capacidad creativa, mejora la memorización y el desarrollo de la abstracción, todo mediante el uso de determinadas melodías, también permite una mayor habilidad verbal y competencia lingüística, así como el desarrollo de la inteligencia matemática, con la cual se ha encontrado relación 
tal como lo afirman Liern y Queralt (2008), se ha demostrado que la música puede influir en el desarrollo de ambos hemisferios del cerebro, así lo expresa Ferrerós (2008).

Otro tipo lo constituye la inteligencia interpersonal, definida como la forma armoniosa de establecer relaciones con otras personas, el desarrollo de esta inteligencia permite tener empatía con los semejantes y en cierta forma predecir comportamientos de las demás personas de acuerdo con sus actitudes y comportamientos en una determinada situación de convivencia. Por su parte, la inteligencia intrapersonal, se define como el autoconocimiento y capacidad para actuar según las creencias y motivaciones personales, incluye una imagen personal precisa que involucra elementos tales como el autocontrol y la autoestima.

Por último, como octava en la lista de esta tipología está la inteligencia naturalista, relacionada con la posibilidad de establecer relaciones afectivas con el entorno natural, los animales, la vegetación, así como brindar apoyo y cuidado a la naturaleza, también se vincula con la capacidad de distinguir formas inanimadas. Es considerada una de la más influyentes en el individuo a nivel evolutivo, ya que determinó la supervivencia en el entorno natural y la capacidad de adaptación y dominio en el hábitat; sin embargo, es poco valorada académicamente (Alabau, 2019).

En Ecuador, a nivel educativo se considera el desarrollo de diversas capacidades en el individuo, así se evidencia en la Ley Orgánica de Educación intercultural, en la cual según el artículo 2, se toman en cuenta los principios de Interaprendizaje y Multiaprendizaje, ambos como instrumentos potenciadores de dichas capacidades mediante la cultura, el deporte, el acceso a la información y sus tecnologías, la comunicación y el conocimiento, para alcanzar niveles de desarrollo tanto personal como colectivo (Presidencia de la República del Ecuador, 2011).

De esta forma, el desarrollo de las inteligencias múltiples conduce a potenciar las capacidades anteriormente descritas, para ello se han usado diferentes recursos y estrategias, entre ellas las tecnologías, donde las TIC se han convertido en herramientas indispensables para la consecución de la actividad educativa, sobre todo en estos tiempos donde la aparición del virus causante de la covid-19 obligó a los gobiernos a nivel mundial a tomar medidas de bioseguridad, que incluyeron el confinamiento de todos los ciudadanos. Las TIC son definidas como:

Herramientas aplicadas para enviar, reproducir, almacenar información, de un lugar a otro; todos estos elementos forman parte de la cultura tecnológica que rodea a la humanidad y con la que debe convivir. Son la base que permitirá disminuir la brecha digital y desarrollar cada vez más, la llamada sociedad de la información y del conocimiento. Las TIC constituyen el conjunto de dispositivos relacionados con las computadoras, internet, telefonía, aplicaciones multimedia (texto, imagen, audio y video) y realidad virtual; tienen como fin mejorar la calidad de vida de las personas. (Quezada y Arrieta, 2019, p. 105).

Para el uso de tecnologías, desde décadas anteriores se han realizado investigaciones que evidencian su efectividad en el campo educativo, al 
respecto, Viñas (2011) estableció un mapa mental donde mostró un conjunto de recursos apoyados en TIC para desarrollar las inteligencias múltiples; de esta forma, para la inteligencia lingüística propone el uso de e-books, enciclopedias y diccionarios virtuales, buscadores y metabuscadores para interpretar la información en la web, redes sociales, programas creadores de podcasts, de historias, poemas, y procesadores de texto.

Entre los recursos mencionados, los e-books o libros electrónicos se han incorporado de tal forma que sólo se transfiere lo impreso a una pantalla digital, sin considerar las experiencias interactivas que ofrecen los recursos tecnológicos y comunicativos. El rápido crecimiento tecnológico ha hecho posible la aparición de varios formatos de lectura, tal como: pdf, txt, html, epub, prc/mobi, eBook, eText, donde su selección se realiza según las necesidades pedagógicas, por ejemplo, para la carrera de Literatura se puede ofrecer una lectura de un texto sin imágenes; sin embargo, para otras tal como por ejemplo artes plásticas, diseño o medicina, sobre todo en el área de anatomía, resulta imprescindible el color y un texto con imágenes. (Medina, 2017). De esta forma, el uso de un e-book además de ayudar a potenciar la inteligencia lingüística también puede hacerlo con la visualespacial.

Según expresa Vanoli (2018), si bien el procesador de texto que más se utiliza es Word entre los programas de Microsoft Office, es importante hacer notar que no es un software libre o gratuito, esta condición abren paso a otras alternativas, como es el uso de Writer del paquete OpenOffice o LibreOffice, o LaTeX, un sistema preciso y potente, pero más complejo a la hora de aleccionar a los estudiantes que recién se inician una carrera profesional; además existen diversos procesadores de texto en la "nube", entre los más reconocidos están los Documentos de Google (Google Docs.) y los Documentos de Word de OneDrive. Estos recursos están permanentemente en actualización y poseen adaptaciones para dispositivos móviles tales como celulares y tabletas.

Con relación a la inteligencialógico-matemática, Viñas (2011) propone el uso de hojas de cálculo, las API como la WebQuest, API matemáticos y ajedrez en línea. Para la inteligencia visualespacial: creadores de modelos 3D, editores de imágenes, líneas de tiempo interactivas, programas de elaboración de gráficos y esquemas, uso de la cámara digital y creadores de presentaciones. Con respecto a la inteligencia corporal-cinética sugiere el uso de programas productores de videos dirigidos a observar y mostrar actividades deportivas y bailes; proyectos de construcción, como por ejemplo robótica; viajes virtuales, uso de cámara de video y visualizaciones. Para la inteligencia musical propone el uso de libros con elementos de audio, web para componer y compartir música, programas editores de sonido, instrumentos musicales virtuales y karaoke.

Para la inteligencia intrapersonal, Viñas (2011) propone la utilización de blogs personal, mapas conceptuales, portafolios multimedia, proyectos con video, software de gestión de tareas y test de personalidad; de la misma forma, para la inteligencia interpersonal, sugiere: el blog de aula, video conferencia, comunidad virtual, redes sociales, juegos colaborativos y presentaciones grupales; de esta forma, según Molinero y Chávez (2019), a través del uso de la herramienta de Google Docs. 
se puede realizar trabajo en equipo favoreciendo el aprendizaje grupal. Por último, para la inteligencia naturalista propone usar grabación de excursiones, instrumentos de exploración digitales y viajes virtuales.

Las TIC, para el desarrollo de las inteligencias múltiples ofrecen una variedad de propuestas, por ejemplo, algunas herramientas con posibilidades de aprovechamiento para trabajar con los estudiantes de manera digital e interactiva son las siguientes: Hojas de cálculo como Excel y OpenOffice Calc., propuestas para aprender a programar como Code, Codecademy, Alice o Scratch, matemáticas interactivas y visuales como Descartes, juegos de lógica como los de Puzzleclopedia, BuscoAcertijos o Acertijos Matemáticos, recursos para crear líneas de tiempo como Timeline o Timerime, herramientas para crear mapas conceptuales como Cmap, Creately, Goconqr o Mindmup, juegos interactivos como los de Vedoque, Matemáticas online, o Little Smart Planet. (AulaPlaneta, 2021b)

Así, setienen como antecedentes, investigaciones que se han encaminado a trabajar sobre este aspecto, a tal efecto está el trabajo de Abrigo, Soto y Treviño (2018), quienes se propusieron estudiar la utilización de las TIC como opción para lograr el desarrollo de las IM planteadas por Howard Gardner en el sector universitario, fue realizada con alumnos de la Universidad Internacional del Ecuador; concluyeron que los aprendices acrecientan sus inteligencias múltiples utilizando recursos tecnológicos como: procesador de textos Word, libros digitales, hojas de cálculo Excel, emplear cámara digital, crear presentaciones, editar videos, realizar proyectos digitales, viajes virtuales, blog personal, mapas conceptuales en programas digitales, software de gestión de tareas, entre otros, además encontraron que existe una escasa utilización de las TIC orientadas al desarrollo de las inteligencias musical, interpersonal y naturalista.

Chalen y Moncada (2018), realizaron una investigación cuyo objetivo fue implementar una estrategia con las inteligencias múltiples aplicando herramientas tecnológicas e interactivas para desarrollar las habilidades y destrezas en los jóvenes que incursionan en el mundo de la programación media de la Facultad de Ciencias Matemáticas y Físicas del Tercer Semestre de la carrera de Ingeniería en Sistemas Computacionales.

En un nivel diferente al universitario se tiene el trabajo presentado por Neira, Del Moral y Fombella (2019), quienes realizaron un estudio cuya finalidad fue determinar la capacidad de una propuesta didáctica apoyada en un entorno con realidad aumentada para propiciar un aprendizaje inmersivo e impulsar las Inteligencias Múltiples en Educación Infantil. Estos autores concluyen que la combinación de actividades apoyadas o no en realidad aumentada, con distintos niveles de acción cognoscitiva, permite estimular globalmente todas las inteligencias.

También se encontraron otras investigaciones relacionadas con el desarrollo de las inteligencias múltiples usando tecnologías tales como las de Viñas (2011), Segarra (2015), Ruiz (2016), GarcíaVélez y Maldonado (2017), Pérez, Álvarez y Guevara (2019), Bravo (2019), Cejas et al. (2020), Moncayo (2020); y, Bravo y García (2020), quienes trabajaron en distintos niveles educativos y demostraron la efectividad del uso de las tecnologías.

Todas las investigaciones mencionadas hacen notar entre sus resultados los beneficios de la 
tecnología para el desarrollo de las inteligencias múltiples, y de la importancia de esta última en los procesos de aprendizaje; sin embargo, en la Universidad de Guayaquil, específicamente en la carrera de Literatura de la Facultad de Filosofía, Letras y Ciencias de la Educación se nota un acentuado desarrollo de la inteligencia lingüística, dejando a un lado la aplicación de recursos para la potenciación de las otras inteligencias propuestas en el modelo de Gardner. Por tal motivo se realizó la presente investigación, la cual tuvo por objetivo determinar el uso de tecnologías para promover el desarrollo de las inteligencias múltiples en los estudiantes la carrera de Literatura de la Facultad de Filosofía, Letras y Ciencias de la Educación.

\section{MÉTODO}

Se realizó una investigación cuantitativa, tipo descriptiva, la cual según su alcance fue exploratoria, siguiendo los planteamientos de Hernández et al. (2014). El diseño asumido fue de campo, no experimental, transversal. De campo porque se observó el fenómeno estudiado tal como se da en su contexto natural, es decir, en el contexto educativo universitario, para posteriormente describirlo. Fue no experimental porque los eventos no fueron provocados intencionalmente. Esto es, no es posible manipular las variables, no se puede influir sobre ellas, porque ya sucedieron, al igual que sus efectos. Fue transeccional o transversal, debido a que se recolectaron datos en un solo momento, en un tiempo único. (Hurtado, 2012).

Se consideró una población de 120 estudiantes inscritos en la carrera de literatura de la Facultad de Filosofía, Letras y Ciencias de la Educación, Universidad de Guayaquil, seleccionando la totalidad de ellos por ser una población finita y de pocos individuos. Se aplicó la técnica de la encuesta, con un cuestionario como instrumento de recolección de datos, apoyado en lo expuesto por Viñas (2011), el cual quedó constituido por 40 proposiciones de respuestas cerradas, con cinco alternativas de selección: siempre $(\mathrm{S})$, casi siempre $(\mathrm{CS})$, algunas veces $(\mathrm{AV})$, casi nunca $(\mathrm{CN})$ y nunca $(\mathrm{N})$; distribuidas en ocho dimensiones correspondientes a cada inteligencia múltiple. Dicho cuestionario fue validado por cinco expertos en educación cuya trayectoria es demostrada en el área del uso de la tecnología, también fue determinada su confiabilidad, obteniendo un valor de 0.83 para el Coeficiente Alfa de Cronbach, el cual es considerado muy alto según Hernández et al. (2014). Fue aplicado a través de Google Forms.

Para presentación de resultados y el análisis de los datos se utilizó la tabla de distribución de frecuencias, con sus respectivos porcentajes. Ya que los datos obtenidos informaron sobre valores concretos que adoptaron cada opción y porcentaje de veces que se repite cada uno de los valores. Para el procesamiento de los datos se utilizó el programa estadístico SPSS 25.

\section{RESULTADOS Y DISCUSIÓN}

A continuación, se presentan los resultados del cuestionario aplicado, el mismo está estructurado en función de las ocho dimensiones, para cumplir con el objetivo de determinar el uso de tecnologías para promover el desarrollo de las inteligencias múltiples en los estudiantes la carrera de Literatura de la Facultad de Filosofía, Letras y Ciencias de la Educación. Para la primera, llamada Inteligencia Lingüística se muestra la Tabla 1. 
Tabla 1. Frecuencias y porcentajes de respuesta de los ítems de la dimensión Inteligencia Lingüística.

\begin{tabular}{|c|c|c|c|c|c|c|c|c|c|c|}
\hline Sus docentes promueven el uso de: & S & $\% \mathrm{~S}$ & CS & $\% \mathrm{CS}$ & AV & $\% \mathrm{AV}$ & $\mathrm{CN}$ & $\% \mathrm{CN}$ & $\mathbf{N}$ & $\% \mathrm{~N}$ \\
\hline $\begin{array}{l}\text { Procesadores de texto (Word, google } \\
\text { docs., otros) }\end{array}$ & 110 & 91.67 & 7 & 5.83 & 3 & 2.50 & 0 & 0.00 & 0 & 0.00 \\
\hline $\begin{array}{l}\text { Programas creadores } \\
\text { poemas o historias, } \\
\text { jumper) }\end{array}$ & 60 & 50.00 & 50 & 41.67 & 10 & 8.33 & 0 & 0.00 & 0 & 0.00 \\
\hline $\begin{array}{l}\text { Programas creadores de podcats y } \\
\text { anotaciones de voz (Audacity) }\end{array}$ & 55 & 45.83 & 43 & 35.83 & 12 & 10.00 & 10 & 8.33 & 0 & 0.00 \\
\hline Enciclopedias y diccionarios en la web & 98 & 81.67 & 14 & 11.67 & 1 & 0.83 & 6 & 5.00 & 1 & 0.83 \\
\hline E-books (ibooks) & 108 & 90.00 & 9 & 7.50 & 2 & 1.67 & 1 & 0.83 & 0 & 0.00 \\
\hline
\end{tabular}

Como se puede observar en la Tabla 1, la mayoría de los estudiantes opinó que sus docentes siempre promueven el uso de los procesadores de texto en un 91.67\%; los programas creadores de historias, poemas o artículos, tales como pixton, story jumper en un 50\%; los programas creadores de podcats y anotaciones de voz en un $45.83 \%$; las enciclopedias y diccionarios en la web en un $81.67 \%$; y, los e-books en un $90 \%$; destacando los primeros como los mayormente usados. Con relación a la segunda dimensión: Inteligencia lógico-matemática se muestra la Tabla 2.

Tabla 2. Frecuencias y porcentajes de respuesta de los ítems de la dimensión Inteligencia lógico-matemática.

\begin{tabular}{|c|c|c|c|c|c|c|c|c|c|c|}
\hline Sus docentes promueven el uso de: & $\mathbf{S}$ & $\% \mathrm{~S}$ & CS & $\% \mathrm{CS}$ & AV & $\% A V$ & $\mathrm{CN}$ & $\% \mathrm{CN}$ & $\mathbf{N}$ & $\% \mathrm{~N}$ \\
\hline Hojas de cálculo (Excel) & 2 & 1.67 & 5 & 4.17 & 6 & 5.00 & 15 & 12.50 & 92 & 76.67 \\
\hline Software estadístico & 0 & 0.00 & 0 & 0.00 & 5 & 4.17 & 12 & 10.00 & 103 & 85.83 \\
\hline Software matemático geogebra & 0 & 0.00 & 0 & 0.00 & 0 & 0.00 & 8 & 6.67 & 112 & 93.33 \\
\hline Webquest & 0 & 0.00 & 0 & 0.00 & 12 & 10.00 & 14 & 11.67 & 94 & 78.33 \\
\hline Ajedrez on line & 0 & 0.00 & 5 & 4.17 & 11 & 9.17 & 20 & 16.67 & 84 & 70.00 \\
\hline
\end{tabular}

Según lo evidenciado en la Tabla 2, la mayoría de los estudiantes opinó que sus docentes nunca promueven el uso de hojas de cálculo, el 76.67\% asílo manifestó; de igual forma sucedió con los softwares estadísticos, donde se ubicaron un $85.83 \%$ de las respuestas; para las API en matemáticas un 93.33\%; los Webquest un 78.33\%; y el ajedrez on line con un $70 \%$. Para la tercera dimensión: Inteligencia visualespacial se muestra la Tabla 3. 
Tabla 3. Frecuencias y porcentajes de respuesta de los ítems de la dimensión visual-espacial.

\begin{tabular}{lcccccccccc}
\hline \multicolumn{1}{c}{ Sus docentes promueven el uso de: } & S & $\%$ S & CS & \%CS & AV & \%AV & CN & \%CN & N & \%N \\
\hline Creadores de modelos 3D (Sketch Up) & 1 & 0.83 & 5 & 4.17 & 8 & 6.67 & 43 & 35.83 & 63 & 52.50 \\
Editores de imágenes (picnick) & 2 & 1.67 & 1 & 0.83 & 9 & 7.50 & 50 & 41.67 & 58 & 48.33 \\
Líneas de tiempo interactivas (tiki-toki) & 30 & 25.00 & 56 & 46.67 & 33 & 27.50 & 1 & 0.83 & 0 & 0.00 \\
Creador de presentaciones (Prezzi) & 35 & 29.17 & 60 & 50.00 & 25 & 20.83 & 0 & 0.00 & 0 & 0.00 \\
Compartir imágenes (Flickr) & 38 & 31.67 & 64 & 53.33 & 17 & 14.17 & 1 & 0.83 & 0 & 0.00 \\
\hline
\end{tabular}

En la Tabla 3 se evidencia que la mayoría de los estudiantes expresó que sus docentes nunca promueven el uso de Creadores de modelos 3D, donde se observa que el $52.50 \%$ así lo manifestó; de igual forma sucedió con los Editores de imágenes, donde se ubicaron un $48.33 \%$ de las respuestas; sin embargo, con relación al uso de Líneas de tiempo interactivas, se tiene que un $46.67 \%$ opino que son usadas casi siempre; igualmente para el Creador de presentaciones se obtuvo un 50\%; y Compartir imágenes un $53.33 \%$. Con respecto a la cuarta dimensión: Inteligencia corporal-cinética se muestra la Tabla 4.

Tabla 4. Frecuencias y porcentajes de respuesta de los ítems de la dimensión corporal-cinética.

\begin{tabular}{lcccccccccc}
\hline \multicolumn{1}{c}{ Sus docentes promueven el uso de: } & $\mathbf{S}$ & $\% \mathrm{~S}$ & $\mathrm{CS}$ & \%CS & $\mathrm{AV}$ & \%AV & $\mathrm{CN}$ & $\% \mathbf{C N}$ & $\mathbf{N}$ & $\% \mathbf{N}$ \\
\hline Proyectos de construcción & 0 & 0.00 & 0 & 0.00 & 2 & 1.67 & 44 & 36.67 & 74 & 61.67 \\
Producción de videos on line & 2 & 1.67 & 22 & 18.33 & 77 & 64.17 & 11 & 9.17 & 8 & 6.67 \\
Viajes virtuales & 0 & 0.00 & 0 & 0.00 & 8 & 6.67 & 14 & 11.67 & 98 & 81.67 \\
Vizualización & 0 & 0.00 & 2 & 1.67 & 4 & 3.33 & 15 & 12.50 & 99 & 82.50 \\
Cámara de vídeo & 75 & 62.50 & 33 & 27.50 & 12 & 10.00 & 0 & 0.00 & 0 & 0.00 \\
\hline
\end{tabular}

Según lo evidenciado en la Tabla 4, la mayoría de los estudiantes opinó que sus docentes nunca promueven el uso de proyectos de construcción, el 61.67\% asílo manifestó; para la producción de videos on line, las respuestas se ubicaron en su mayoría en algunas veces, con un $64.17 \%$ de respuestas; sin embargo, con respecto al uso de viajes virtuales, y la visualización, la mayoría de las respuestas se encuentran en la alternativa nunca, con un $81.67 \%$ y $82.50 \%$ respectivamente; mientras que el uso de la cámara de vídeo se ubica en la alternativa siempre, con un $62.50 \%$ de las respuestas. Los resultados correspondientes a la quinta dimensión: Inteligencia musical se muestra la Tabla 5. 
Tabla 5. Frecuencias y porcentajes de respuesta de los ítems de la dimensión musical.

\begin{tabular}{lcccccccccc}
\hline \multicolumn{1}{c}{ Sus docentes promueven el uso de: } & $\mathbf{S}$ & $\% \mathbf{S}$ & $\mathbf{C S}$ & $\% \mathbf{C S}$ & $\mathrm{AV}$ & \%AV & $\mathbf{C N}$ & $\% \mathbf{C N}$ & $\mathbf{N}$ & $\% \mathbf{N}$ \\
\hline Libros con elementos de audio & 0 & 0.00 & 20 & 16.67 & 48 & 40.00 & 51 & 42.50 & 1 & 0.83 \\
Webs para compartir música & 3 & 2.50 & 18 & 15.00 & 56 & 46.67 & 43 & 35.83 & 0 & 0.00 \\
Editores de sonido & 0 & 0.00 & 15 & 12.50 & 12 & 10.00 & 84 & 70.00 & 9 & 7.50 \\
Karaoke & 0 & 0.00 & 2 & 1.67 & 8 & 6.67 & 40 & 33.33 & 70 & 58.33 \\
Instrumentos musicales virtuales & 0 & 0.00 & 0 & 0.00 & 3 & 2.50 & 17 & 14.17 & 100 & 83.33 \\
\hline
\end{tabular}

Como puede observarse en la Tabla 5, la mayoría de los estudiantes opinó que sus docentes casi nunca promueven el uso de Libros con elementos de audio, el $42.50 \%$ así lo manifestó; con respecto al uso de las webs para compartir música, las respuestas se ubicaron en su mayoría en algunas veces, con un 46.67\%; con relación al uso de editores de sonido, la mayoría de las respuestas se encuentran en la alternativa casi nunca, con un $70 \%$; mientras que el uso de karaoke e instrumentos musicales virtuales se ubicaron en la alternativa nunca, con un $58.33 \%$ y $83.33 \%$ de las respuestas respectivamente. Con respecto a la sexta dimensión: Inteligencia Intrapersonal se muestra la Tabla 6.

Tabla 6. Frecuencias y porcentajes de respuesta de los ítems de la dimensión Intrapersonal.

\begin{tabular}{lcccccccccc}
\hline \multicolumn{1}{c}{ Sus docentes promueven el uso de: } & S & $\%$ S & CS & \%CS & AV & \%AV & CN & \%CN & N & \%N \\
\hline Blog personal & 12 & 10.00 & 51 & 42.50 & 25 & 20.83 & 15 & 12.50 & 17 & 14.17 \\
Portafolio multimedia & 2 & 1.67 & 66 & 55.00 & 22 & 18.33 & 16 & 13.33 & 14 & 11.67 \\
Software de gestión de tareas & 9 & 7.50 & 63 & 52.50 & 19 & 15.83 & 15 & 12.50 & 14 & 11.67 \\
Test de personalidad & 0 & 0.00 & 0 & 0.00 & 1 & 0.83 & 0 & 0.00 & 119 & 99.17 \\
Mapas conceptuales & 98 & 81.67 & 13 & 10.83 & 9 & 7.50 & 0 & 0.00 & 0 & 0.00 \\
\hline
\end{tabular}

Para la dimensión intrapersonal, puede observarse en la Tabla 6 que la mayoría de los estudiantes expresó que sus docentes casi siempre promueven el uso de Blog personal, con un $42.50 \%$, el portafolio multimedia con un 55\% y el Software de gestión de tareas con un $52.50 \%$; con respecto al uso del test de personalidad, las respuestas se ubicaron en su mayoría en nunca, con un $99.17 \%$; mientras que el uso de mapas conceptuales se ubicó en la alternativa siempre, con un $81.67 \%$. Para la séptima dimensión: Inteligencia Interpersonal se muestra la Tabla 7. 
Tabla 7. Frecuencias y porcentajes de respuesta de los ítems de la dimensión Interpersonal.

\begin{tabular}{lcccccccccc}
\hline \multicolumn{1}{c}{ Sus docentes promueven el uso de: } & $\mathbf{S}$ & $\% \mathbf{S}$ & $\mathrm{CS}$ & \%CS & $\mathrm{AV}$ & \%AV & $\mathrm{CN}$ & $\% \mathbf{C N}$ & $\mathbf{N}$ & $\% \mathbf{N}$ \\
\hline Presentaciones grupales & 79 & 65.83 & 21 & 17.50 & 16 & 13.33 & 3 & 2.50 & 1 & 0.83 \\
Juegos colaborativos & 8 & 6.67 & 18 & 15.00 & 21 & 17.50 & 72 & 60.00 & 1 & 0.83 \\
Redes sociales & 95 & 79.17 & 11 & 9.17 & 9 & 7.50 & 4 & 3.33 & 1 & 0.83 \\
Comunidad virtual & 0 & 0.00 & 7 & 5.83 & 19 & 15.83 & 82 & 68.33 & 12 & 10.00 \\
Video conferencias & 91 & 75.83 & 13 & 10.83 & 9 & 7.50 & 7 & 5.83 & 0 & 0.00 \\
\hline
\end{tabular}

Como puede observarse en la Tabla 7, la mayoría de los estudiantes opinó que sus docentes siempre promueven el uso de Presentaciones grupales, Redes sociales y Video conferencias, con un $65.83 \%$, $79.17 \%$ y $75.83 \%$ respectivamente; sin embargo, el uso de Juegos colaborativos y comunidad virtual se ubicaron en la alternativa nunca, con un $60 \%$ y $68.33 \%$ de las respuestas respectivamente. Con respecto a la octava dimensión: Inteligencia Naturalista se muestra la Tabla 8.

Tabla 8. Frecuencias y porcentajes de respuesta de los ítems de la dimensión Naturalista.

\begin{tabular}{lcccccccccc}
\hline \multicolumn{1}{c}{ Sus docentes promueven el uso de: } & $\mathbf{S}$ & $\% \mathbf{S}$ & $\mathbf{C S}$ & $\% \mathbf{C S}$ & $\mathrm{AV}$ & \%AV & $\mathbf{C N}$ & $\% \mathbf{C N}$ & $\mathbf{N}$ & $\% \mathbf{N}$ \\
\hline Excursiones virtuales & 0 & 0.00 & 0 & 0.00 & 3 & 2.50 & 11 & 9.17 & 106 & 88.33 \\
Foros conservacionistas & 0 & 0.00 & 2 & 1.67 & 5 & 4.17 & 8 & 6.67 & 105 & 87.50 \\
Herramientas de observación virtuales & 0 & 0.00 & 0 & 0.00 & 0 & 0.00 & 3 & 2.50 & 117 & 97.50 \\
Juegos virtuales sobre conservación ambiental & 0 & 0.00 & 0 & 0.00 & 0 & 0.00 & 0 & 0.00 & 120 & 100.0 \\
Proyectos sobre la naturaleza & 3 & 2.50 & 2 & 1.67 & 0 & 0.00 & 15 & 12.50 & 100 & 83.33 \\
\hline
\end{tabular}

Para la dimensión naturalista, puede observarse en la Tabla 8 que la mayoría de los estudiantes expresó que sus docentes nunca promueven el uso de excursiones virtuales, con un $83.3350 \%$, la participación en Foros conservacionistas, con un 87.50\%, el uso de Herramientas de observación virtuales, con un 97.50\%; los Juegos virtuales sobre conservación ambiental, con un $100 \%$ y la realización de Proyectos sobre la naturaleza, con un $83.33 \%$

A continuación, se muestra la Figura 1 un resumen de la mayoría de las respuestas en cada una de las dimensiones. 


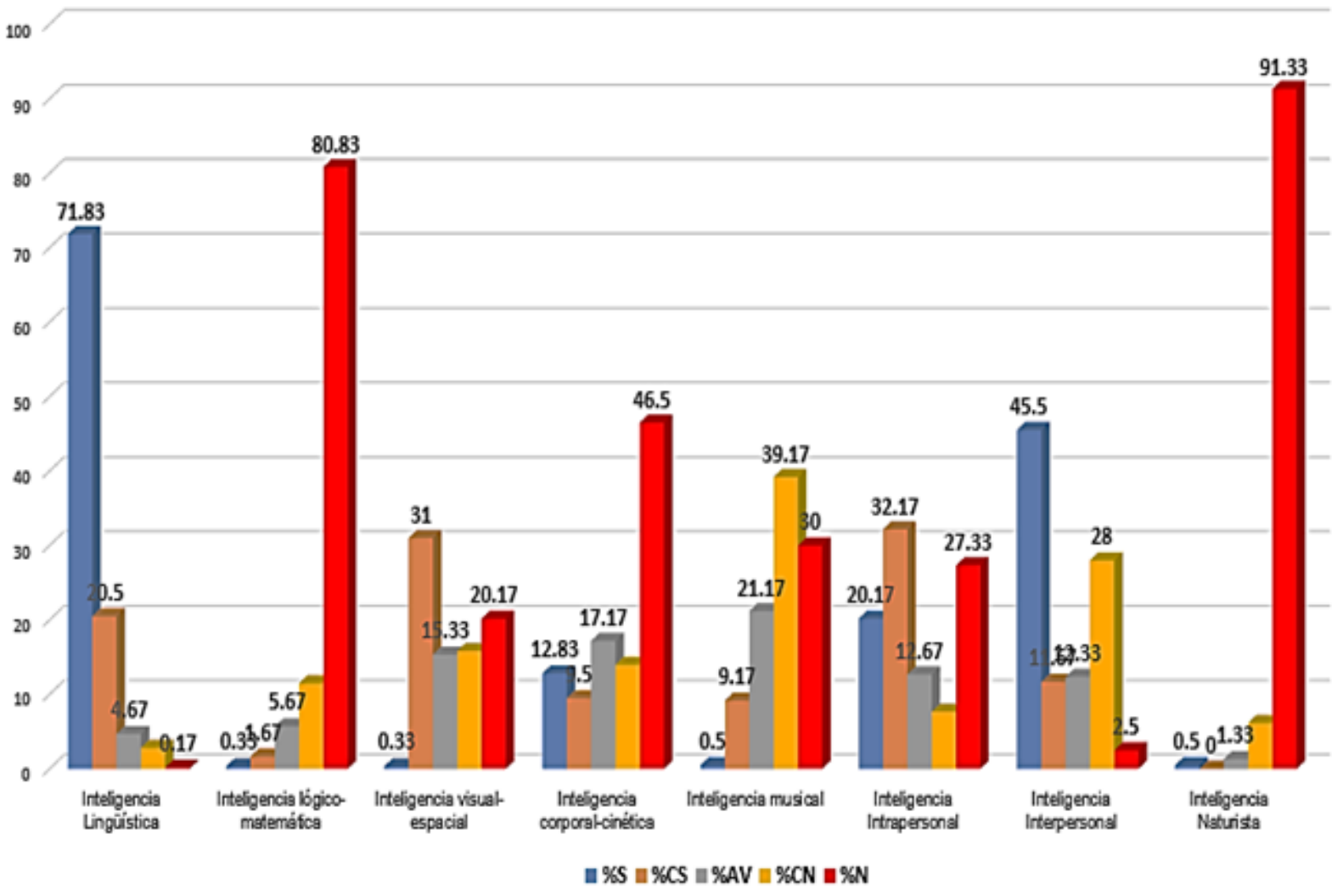

Figura 1. Porcentajes de respuesta de las dimensiones estudiadas.

Como se evidencia en la Figura 1, la tendencia para la promoción del uso de tecnología para el desarrollo de la inteligencia lingüística es hacia la alternativa siempre, con un $71,83 \%$ de las respuestas de los estudiantes, para la lógico-matemática se obtuvo un $80.83 \%$ en la alternativa nunca, la visualespacial se inclinó hacia casi siempre con un $31 \%$, la corporal-cinética se colocó en la alternativa nunca con un $46.50 \%$, la musical en casi nunca con un $39.17 \%$, la intrapersonal se posicionó en casi siempre con $32.17 \%$, la interpersonal con $45.50 \%$ en siempre, y la naturalista fue una de las más altas, con un $91.33 \%$ en la alternativa nunca.

\section{Discusión}

Tal como se evidenció en los resultados, según las respuestas de la mayoría de los estudiantes, sus docentes promueven el uso de tecnologías que desarrollan la inteligencia lingüística, tomando en consideración que para Gardner (1994) está inteligencia es la competencia intelectual más compartida por los seres humanos, para este autor, el dominio del lenguaje oral y escrito involucra procesos mentales intelectuales complejos que lo convierten en uno de los aspectos más trabajados y más usados por los individuos. 
Estos resultados coinciden con los de Abrigo, Soto y Treviño (2018), quienes consiguieron que los estudiantes expresaron con frecuencia, que las tecnologías impulsan la inteligencia lingüística, al utilizar Word, revistas digitales, leer libros ubicados en la web, redactar e-mails, emplear programas de podcasts y anotaciones de voz. También concuerdan con los resultados de la investigación de Molinero y Chávez (2019), quienes encontraron que, a pesar del tiempo transcurrido, los programas de Microsoft Office siguen siendo los preferidos por los aprendices, donde Word es el procesador más usado. Es importante destacar que estos resultados son extraídos de las respuestas de estudiantes la carrera de literatura, quienes, por la naturaleza de su formación académica, hacen mayor énfasis en el desarrollo de competencias comunicativas y literarias.

Con relación al desarrollo de la inteligencia lógico-matemática, todas las respuestas en su mayoría están ubicadas en la alternativa nunca; esto es, los docentes no están promoviendo el uso de tecnologías apropiadas para esta, estos resultados no contemplan lo encontrado por Abrigo, Soto y Treviño (2018), quienes expusieron que la mayoría de los estudiantes participantes de su investigación expresaron en alguna ocasión, y con frecuencia; que el empleo de tecnologías tales como hojas de cálculo Excel, programas estadísticos para tabulación de datos y software matemático, habían sido beneficiosas para el desarrollo de su inteligencia lógico-matemática.

Las tecnologías para promover el desarrollo de la Inteligencia visual-espacial son usadas en parte; es decir, los estudiantes opinaron que sus docentes si hacen uso de herramientas para elaborar Líneas de tiempo interactivas, de software creadores de presentaciones y de recursos para compartir imágenes; sin embargo, en su mayoría nunca promovieron el uso de creadores de modelos $3 \mathrm{D}$ ni de programas para editar imágenes, esto concuerda con el uso de recursos necesarios para la formación del perfil profesional de los estudiantes de la carrera en literatura; sin embargo, no se considera la opinión de Armstrong (2000), quien manifiesta que conforme aumenta la inclusión de las tecnologías información y comunicación a la cotidianidad, puede progresar el valor asignado a una inteligencia espacial muy desarrollada.

La inteligencia corporal-cinética, según los resultados evidenciados, en su mayoría nunca se promueven tecnologías que ayuden a su desarrollo, a excepción del uso de la cámara de vídeo, lo cual se hace siempre y la producción de videos on line algunas veces. Es decir, estos docentes descuidan el desarrollo de esta inteligencia, lo cual concuerda con el resultado de Segarra (2015), quien encontró que los educadores se enfocan en desarrollar actividades curriculares propias de su asignatura, sin considerar otras estrategias y recursos para promover la consolidación de esta inteligencia.

Con respecto a los resultados de la dimensión inteligencia musical se obtuvo que la mayoría de los docentes nunca o casi nunca posibilitó el uso de tecnologías que desarrollaran este tipo de inteligencia, sin considerar lo planteado por García-Vélez y Maldonado (2017) quienes expresan que la utilización de la música en el acto educativo posibilita un proceso de enseñanza y aprendizaje con acceso a distintas destrezas importantes, tales como la empatía y la autorregulación emocional, las cuales permiten establecer una vinculación efectiva y afectiva con el contexto. 
Para la inteligencia intrapersonal se encontró que la mayoría de los docentes casi siempre y siempre promueven el uso de tecnologías para su desarrollo, este resultado apoya al encontrado por Abrigo, Soto y Treviño (2018), quienes manifestaron que los estudiantes de su investigación consideran a las tecnologías como herramientas útiles para el desarrollo de esta inteligencia, al crear un blog personal, realizar mapas conceptuales en programas digitales, o utilizar un software de gestión de tareas.

El desarrollo de la inteligencia interpersonal, según las respuestas de los estudiantes, fue trabajado por sus docentes mediante la siempre utilización de presentaciones grupales, redes sociales y video conferencias; sin embargo, casi nunca usan juegos colaborativos y comunidades virtuales; esto último puede ser a causa de que se está trabajando a nivel universitario, donde las estrategias basadas en juegos son poco frecuentes. Con respeto al uso de redes sociales, estos resultados están en consonancia con la opinión de Ruiz (2016), quien manifestó que su uso favorece la interacción grupal en línea, compartir contenidos, y realizar proyectos colaborativos.

Para la inteligencia naturalista se obtuvo que la mayoría de los estudiantes manifestó que sus docentes nunca promovían el desarrollo de esta, debido a que no utilizan tecnologías tales como Excursiones virtuales, Foros conservacionistas, Herramientas de observación como microscopios, prismáticos o telescopios virtuales, Juegos virtuales sobre conservación ambiental, ni Proyectos sobre la naturaleza. De esta forma, estos resultados se relacionas con lo expresado por Alabau (2019), quien manifestó que esta es una habilidad poco valorada académicamente; además se tiene que no muestran coherencia con los planteamientos del modelo ecológico de la Universidad de Guayaquil (Larrea y Montalván, 2016) donde se expresa que el sujeto cognoscente debe tener su autonomía dirigida a la interacción con el entorno social y natural, donde prevalezca un diálogo ecológico de saberes.

\section{CONCLUSIONES}

El uso de tecnologías en educación a nivel superior representa una oportunidad para el desarrollo del potencial pleno de los estudiantes, los recursos educativos apoyados en tecnologías en los últimos años se han convertido en la base fundamental para el proceso de enseñanza aprendizaje; estas al ser utilizadas de forma eficiente por los docentes pueden potenciar el desarrollo de las diferentes inteligencias múltiples de los estudiantes.

En esta investigación se determinó el uso de tecnologías para promover el desarrollo de las inteligencias múltiples en los estudiantes la carrera de Literatura de la Facultad de Filosofía, Letras y Ciencias de la Educación. Al respecto se concluye que los docentes de la carrera de Literatura promueven siempre el uso de tecnologías para el desarrollo de la inteligencia lingüística e interpersonal; medianamente, para la intrapersonal y la visual-espacial; sin embargo, se evidenciaron deficiencias en cuanto a la promoción y uso de tecnologías para desarrollar las inteligencias lógico-matemática, corporal-cinética, musical y naturalista.

Se hace necesario un cambio en la praxis educativa de tal forma que permita el aprovechamiento, aplicabilidad y usabilidad de las 
herramientas tecnológicas, para que potencien las inteligencias múltiples, esto debe considerar que el modelo de IM de Gardner contempla que se puede aprender por diferentes vías, lo que posibilita el perfeccionamiento de diferentes capacidades que permiten el desarrollo holístico del ser humano, respondiendo a las necesidades de Ecuador; por tanto, se recomienda diseñar y facilitar un plan de formación y capacitación para docentes universitarios con relación al uso de tecnologías que permitan el desarrollo de las inteligencias múltiples por parte de sus estudiantes.

\section{REFERENCIAS}

Abrigo, I., Soto, J. y Treviño, I. (2018). Recursos TIC'S: una alternativa para desarrollar las inteligencias múltiples. INNOVA Research Journal, 3(4), 15-24. DOI: https://doi. org/10.33890/innova.v3.n4.2018.468

Alabau, I. (3 de octubre de 2019). Inteligencia naturalista: qué es, características, ejemplos y actividades. https://www.psicologiaonline.com/inteligencia-naturalista-que-escaracteristicas-ejemplos-y-actividades-4723. html

Armstrong, T. (2000). Inteligencias múltiples en el aula. 2. edición ampliada y revisada. Paidós.

AulaPlaneta. (2021a). Cómo y porqué desarrollar la inteligencia musical de tus alumnos. https://www.aulaplaneta.com/2019/04/10/ educacion-y-tic/como-y-porque-desarrollar-lainteligencia-musical-de-tus-alumnos/

AulaPlaneta. (2021b). Cómo trabajar en clase la inteligencia lógico-matemática [Infografía]. https://www.aulaplaneta.com/2016/05/24/ recursos-tic/como-trabajar-en-clase-lainteligencia-logico-matematica-infografia/

Bravo, A. y García, C. (2020). Flipped classroom con PowToon para desarrollar inteligencias múltiples. Revista Dominio de las Ciencias, 6(3), 04-25. DOI: http://dx.doi.org/10.23857/ dc.v6i3.1271
Bravo, O. (2019). Las inteligencias múltiples en los procesos de la actualización de conocimientos de los neos licenciados. En 5to Congreso internacional de ciencias pedagógicas de Ecuador. 13 al 11 de abril de 2019, Instituto Superior Tecnológico Bolivariano de Tecnología, Guayaquil, Ecuador. Pp. 572-580

Cejas, M., Lozada, B., Urrego, A., Mendoza, D, y Rivas, G. (2020). La irrupción de las tecnologías de la información y la comunicación (TIC), un reto en la gestión de las competencias digitales de los profesores universitarios en el Ecuador. Revista lbérica de Sistemas y Tecnologías de Información, (37), 132-148. DOI: 10.17013/ risti.37.131-148

Chalen, H. y Moncada, Y. (2018). Desarrollo de habilidades y destrezas en la programación media con las inteligencias múltiples a través de herramientas interactivas. [Trabajo de grado]. https://n9.cl/t0xlz

Ferrerós, M. (2008). Inteligencia musical. Estimula el desarrollo de tu hijo a través de la música. Libros cúpula

García-Vélez, T.y Maldonado, A. (2017). Reflexiones sobre la inteligencia musical. Revista Española de Pedagogía, 75(268), 451-461. DOI: https:// doi.org/10.22550/REP75-3-2017-08

Gardner, H. (1997). Arte, mente y cerebro. Una aproximación cognitiva a la creatividad. Séptima edición. Paidós

Gardner, H. (1994). Estructuras de la mente: La Teoría de las Inteligencias Múltiples. Segunda Edición. Fondo de Cultura Económica

Hernández, R.; Fernández, C. y Baptista, M. (2014). Metodología de la Investigación. Sexta edición. McGraw-Hill Education

Hurtado, J. (2012). Metodología de la Investigación. Edición Quirón.

Larrea, E. y Montalván, M. (2016). Modelo Educativo Ecológico de la Universidad de Guayaquil. Universidad de Guayaquil. https:// www.ug.edu.ec/modelo-educativo-ecologicode-la-universidad-de-guayaquil/ 
Liern, V. y Queralt, T. (2008). Música y matemáticas. La armonía de los números. Servicio de publicaciones de la federación española de sociedades de profesores de matemáticas

Medina, R. (2017). Uso del eBook en el aula. Un medio introductorio en el manejo de las tecnologías emergentes. Revista de Investigación Educativa de la Escuela de Graduados en Educación, 8(15). http://rieege.mx/index.php/ rieege/article/view/183/325

Molinero, M. y Chávez, U. (2019). Herramientas tecnológicas en el proceso de enseñanzaaprendizaje en estudiantes de educación superior. RIDE. Revista Iberoamericana para la Investigación y el Desarrollo Educativo, 10(19). https://doi.org/10.23913/ride.v10i19.494

Moncayo, B. (2020). Las inteligencias múltiples en el proceso de enseñanza aprendizaje de biología en el Bachillerato General Unificado de la Unidad Educativa Particular Ecuatoriano Suizo, 20192020. [Trabajo de grado]. http://www.dspace. uce.edu.ec/handle/25000/21441

Neira, M., Del Moral, M. y Fombella, I. (2019). Aprendizaje inmersivo y desarrollo de las inteligencias múltiples en Educación Infantil a partir de un entorno interactivo con realidad aumentada. Magister 31(2), 1-8. https://www. unioviedo.es/reunido/index.php/MSG

Pérez, H., Álvarez, A. y Guevara, C. (2019). Dominio de habilidades del pensamiento computacional en los estudiantes del Instituto Tecnológico Superior Sucre de Quito - Ecuador. Revista Interuniversitaria de Investigación en Tecnología Educativa (RIITE), (7), 48-60. DOI: http://dx.doi.org/10.6018/riite.394221
Presidencia de la República del Ecuador. (2011). Ley Orgánica de Educación Intercultural. Registro Oficial Suplemento 417 de 31-mar.-2011. Quito, Ecuador

Quezada, J. y Arrieta, X. (2019). Aplicación de la plataforma Dokeos para fortalecer el proceso de enseñanza y aprendizaje en ciencias naturales. Revista Encuentro Educacional. 26(1), 102-122. https://produccioncientificaluz.org/index.php/ encuentro/article/view/34603

Ruiz, C. (2016). Redes Sociales y Educación Universitaria. Paradigma, XXXVII(1), 232-256. http://ve.scielo.org/pdf/pdg/v37n1/art12.pdf

Segarra, E. (2015). La inteligencia kinestésica y el desarrollo motriz fino de los estudiantes de tercer año de la Unidad Educativa Huachi Grande de la Ciudad de Ambato. [Trabajo de grado]. http://repositorio.uta.edu.ec/ handle/123456789/19055

Vanoli, V. (2018). Procesador de Textos: un tema relevante y desatendido en la Enseñanza. Revista de la Facultad de Ciencias Exactas, Físicas y Naturales, 5(1), 85-85. https://revistas.unc.edu. ar/index.php/FCEFyN/article/view/18039

Viñas, M. (29 de octubre de 2011). Mapa mental: Recursos tic para desarrollar las inteligencias múltiples de Howard Gardner. Academy TotemGuard Digital. https://www.totemguard. com/aulatotem/2011/10/mapa-conceptualrecursos-tic-para-desarrollar-las-inteligenciasmultiples-de-howard-gardner/ 